\title{
Long non-coding RNA AB007962 is downregulated in gastric cancer and associated with poor prognosis
}

\author{
JIA-JUN WANG ${ }^{*}$, YU-CHONG YANG ${ }^{*}$, YONG-XI SONG, PENG GAO, \\ JING-XU SUN, XIAO-WAN CHEN, BIN MA and ZHEN-NING WANG \\ Department of Surgical Oncology and General Surgery, The First Hospital of \\ China Medical University, Shenyang, Liaoning 110001, P.R. China
}

Received November 27, 2015; Accepted June 18, 2018

DOI: $10.3892 / \mathrm{ol} .2018 .9169$

\begin{abstract}
A number of previous studies have reported that numerous long non-coding RNAs (lncRNAs) are dysregulated in gastric cancer (GC) and are involved in a series of biological and pathological processes. Total RNA was extracted from the cancerous tissues and matched normal adjacent tissues (NATs) of 96 patients with GC. The expression level of AB007962, a novel lncRNA, was determined by reverse transcription-quantitative polymerase chain reaction. The association between AB007962 expression levels and clinicopathological features were analyzed. Kaplan-Meier curves were also constructed in order to evaluate prognosis. Finally, publicly accessible data from The Cancer Genome Atlas was used to further verify the expression levels and clinical significance of AB007962. In conclusion, it was determined that the expression level of AB007962 was significantly reduced, compared with matched NATs in GC tissues $(\mathrm{P}=0.003)$. Survival analysis indicated that patients with intestinal-type $\mathrm{GC}$ with a reduced expression of AB007962 had a reduced prognosis, compared with those with an increased expression. AB007962 may be involved in the progression of GC and act as a novel prognostic biomarker for patients with GC, particularly in intestinal-type GC.
\end{abstract}

\section{Introduction}

Gastric cancer (GC) is the fourth most common malignancy and causes the second most cancer-associated mortalities globally in 2010, particularly in East Asia $(1,2)$. The majority

Correspondence to: Dr Zhen-Ning Wang, Department of Surgical Oncology and General Surgery, The First Hospital of China Medical University, 155 North Nanjing Street, Shenyang, Liaoning 110001, P.R. China

E-mail: josieon826@sina.cn

*Contributed equally

Key words: long non-coding RNA, AB007962, The Cancer Genome Atlas, reverse transcription-quantitative polymerase chain reaction, prognosis, intestinal-type gastric cancer of GC cases are diagnosed at an advanced stage, which notably contributes to the poor prognosis associated with the disease (3); therefore, it is important to determine novel effective biomarkers, in order to improve the diagnosis and medical treatment of GC.

Long non-coding RNAs (lncRNAs) are transcribed RNA sequences with a length $>200$ nucleotides (nt) and no protein-encoding ability (4). A number of previous studies have reported that numerous lncRNAs are dysregulated in a series of cancer types, including GC, and that they serve as biomarkers for GC diagnosis and prognosis of patients with GC (5-8). For example, HOX antisense intergenic RNA (HOTAIR) was one of the earliest lncRNAs studied. It is upregulated in GC, compared with normal adjacent tissues (NATs), and its expression is a significant predictor of poor prognosis (9). Endo et al (10) reported similar results in diffuse-type GC. The lncRNA H19 has attracted much attention in recent years and the expression levels of H19 in GC tissues are significantly increased compared with NATs, and its expression is notably associated with poor prognosis of patients with GC (5,11-13). However, the role of lncRNAs in GC remains unknown and further research is required.

In the present study, the focus was on the lncRNA AB007962, which is located on chromosome 1 and has a transcript length of 5,053 nt (http://genome.ucsc.edu/cgi-bin/hgGene?hgg_ gene=uc001eso.1\&db=hg19). Its expression was analyzed in GC tissues, and the association between its expression and the clinicopathological features and prognosis of patients with GC was assessed. Furthermore, a cohort of patients with GC from The First Hospital of China Medical University (Shenyang, China) and a cohort from The Cancer Genome Atlas (TCGA) were reviewed, in order to assess the clinical utility of AB007962 expression levels.

\section{Materials and methods}

Specimens and clinical data collection. All of the 96 paired human GC tissues and matched NAT samples were obtained from patients who underwent radical resection of GC at The First Hospital of China Medical University between May 2009 and July 2010. The median age of the 96 patients with GC was 62 years (range, 30-81 years), with 70 males accounting for $72.9 \%$ and 26 females accounting for $27.1 \%$ of the cohort. 
Furthermore, 77 patients $(80.2 \%)$ had lymph node metastasis (pN1-N3) and all of the 96 patients completed follow-up. The matched NAT samples were collected from tissues that were located $\geq 5 \mathrm{~cm}$ from the edge of the tumor. All of the samples were frozen immediately in liquid nitrogen and stored at $-80^{\circ} \mathrm{C}$ until use. Two pathologists from The First Hospital of China Medical University (Shenyang, China) histopathologically confirmed each sample taken for diagnosis of GC. In addition, there was no radiotherapy or chemotherapy prior to resection. The histological grade of the samples was staged by following the standards set by the World Health Organization (14). The tumor stage was also evaluated on the basis of the 7th edition of the Tumor-Node-Metastasis (TNM) staging system (15). Overall survival (OS) was defined as the interval between the date of surgery and the date of mortality or the last follow-up examination. Disease-free survival (DFS) was defined as the interval between the date of surgery and the date of recovery from disease or the last follow-up examination.

Ethical approval of the study protocol. The present study was performed according to the principles expressed in The Declaration of Helsinki. Tissue samples were collected once written informed consent was obtained from all 96 patients, and following institutional ethical guidelines that were reviewed and approved by the Research Ethics Committee of China Medical University (Shenyang, China).

$R N A$ isolation and reverse transcription-quantitative polymerase chain reaction ( $R T-q P C R)$. According to the manufacturer's protocols, total RNA was isolated from frozen specimens of tumor and NATs using TRIzol ${ }^{\circledR}$ (Invitrogen; Thermo Fisher Scientific, Inc., Waltham, MA, USA). The purity of the total RNA was verified by ultraviolet (UV) spectrophotometry (2.0>A260/A280>1.8) using a NanoPhotometer UV/Vis spectrophotometer(Implen GmbH,Münich, Germany). Using a PrimeScript RT reagent kit (Takara Biotechnology Co., Ltd., Dalian, China), RNA was reverse transcribed into cDNA for qPCR according to the manufacturer's protocol. For qPCR, according to the manufacturer's protocol, the $25 \mu \mathrm{l}$ final volume contained 12.5 $\mu 12 \mathrm{X} \mathrm{SYBR}^{\circledR}$ Premix Ex Taq ${ }^{\mathrm{TM}}$ (Takara Biotechnology Co., Ltd.), $0.5 \mu \mathrm{l}$ forward and reverse primers each $(10 \mu \mathrm{M}), 9.5 \mu \mathrm{l}$ nuclease-free water, and $2 \mu$ d diluted of the reverse transcribed product The primers (Sangon Biotech Co., Ltd., Shanghai, China) used in the present study were as follows: GAPDH forward, 5'-CGGATTTGGTCGTATTGG G-3' and reverse, 5'-CTGGAAGATGGTGATGGGATT-3'; AB007962 forward, 5'-TGTGCTCACGATGTTGAATG-3' and reverse, 5'-AATGGAAATGGGACATGGAC-3'. The reaction was incubated in a 96-well optical plate and amplified for: 1 cycle of $95^{\circ} \mathrm{C}$ for $30 \mathrm{sec}$; and 45 cycles of $95^{\circ} \mathrm{C}$ for $5 \mathrm{sec}$ and $60^{\circ} \mathrm{C}$ for $30 \mathrm{sec}$. Relative expression of AB007962 was normalized to GAPDH and calculated using the $2^{-\Delta \Delta C q}$ method (16). $\Delta \mathrm{C}_{\mathrm{q}}$ is the difference in the $\mathrm{C}_{\mathrm{q}}$ value between the target and endogenous reference $\left(\Delta \mathrm{C}_{\mathrm{q}}=\mathrm{C}_{\mathrm{q} \text { IncRNA }}-\mathrm{C}_{\mathrm{q} \text { GAPDH }}\right)$. $\Delta \Delta \mathrm{C}_{\mathrm{q}}$ is the difference in $\Delta \mathrm{C}_{\mathrm{q}}$ values between the tumor cells and the control $\left(\Delta \Delta \mathrm{C}_{\mathrm{q}}=\Delta \mathrm{C}_{\mathrm{q} \text { tumor IncRNA }}-\Delta \mathrm{C}_{\mathrm{qNATs} \text { IncRNA }}\right)$.

TCGA gene expression data. The expression of AB007962 in human gastric specimens was analyzed using publicly available TCGA Illumina RNA-Seq datasets (http://cancergenome.

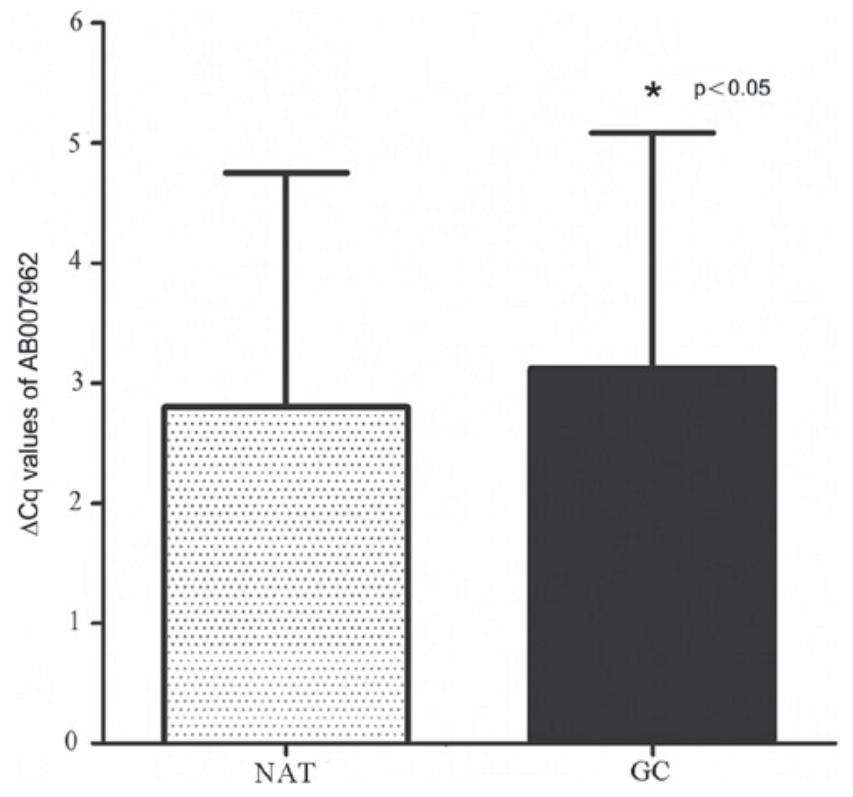

Figure 1. AB007962 is primarily down-regulated in GC tissues, compared with NATs ( $\triangle$ Cq of AB007962). GC, gastric cancer; NATs, normal adjacent tissues.

nih.gov) of 274 patients with GC, including their clinical parameters and follow-up data. The present study used the reads per kilobase of exon per million reads mapped (RPKM) value to represent the expression levels of AB007962 for each patient from TCGA datasets (17). Amongst these, matched tumor and non-tumor specimens were available for 29 patients with GC.

Statistical analysis. Statistical analysis was performed using the SPSS 21.0 software package (IBM Corp., Armonk, NY, USA). A paired Student's t-test was used to evaluate the difference in AB007962 expression between tumor tissues and NATs and the expression levels from TCGA datasets between groups were calculated by a nonparametric test (Wilcoxon test). The association between AB007962 expression and clinicopathological factors was analyzed using the Mann-Whitney U test for two groups and the Kruskal-Wallis test for three or more groups. Survival curves were evaluated by the Kaplan-Meier method and the log-rank test was used to determine whether there was a statistically significant difference between survival curves. Cox proportional hazards analysis was performed to calculate the hazard ratio (HR) and the $95 \%$ confidence interval (CI) to estimate the association between AB007962 expression level and OS and DFS survival time. $\mathrm{P}<0.05$ was considered to indicate a statistically significant difference.

\section{Results}

Expression of AB007962 is downregulated in GC tissues. The expression level of AB007962 in 96 paired cancerous and matched NATs was examined by RT-qPCR. The results demonstrated that AB007962 expression levels were reduced in cancerous tissues, compared with matched NATs (paired Student's t-test; $\mathrm{P}<0.05$; Fig. 1). Furthermore, the AB007962 expression levels in 58/96 (60.4\%) GC tissues were downregulated, compared with matched NATs. The AB007962 
A

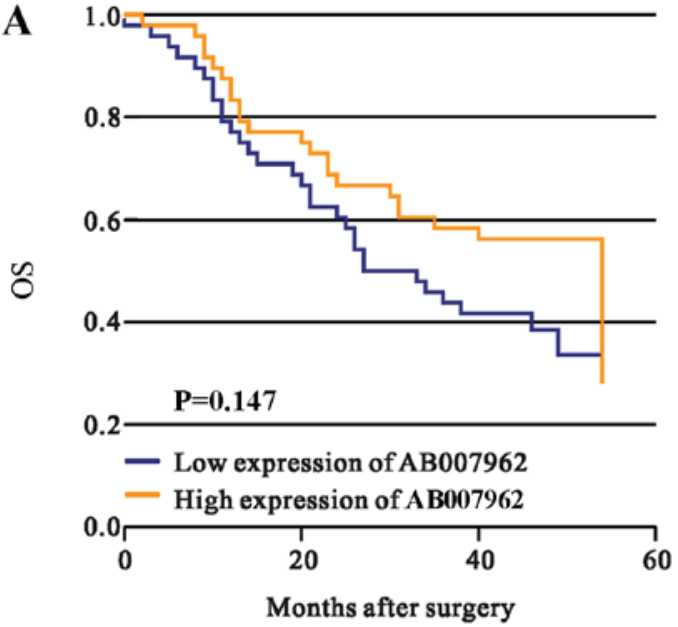

C

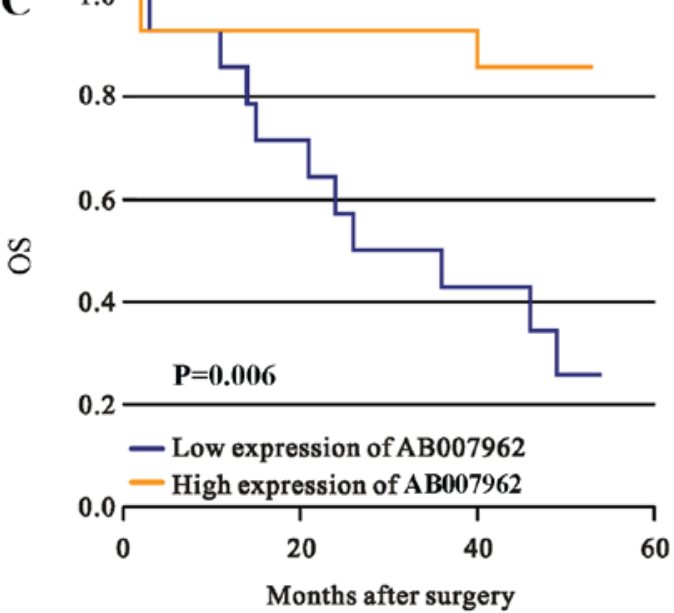

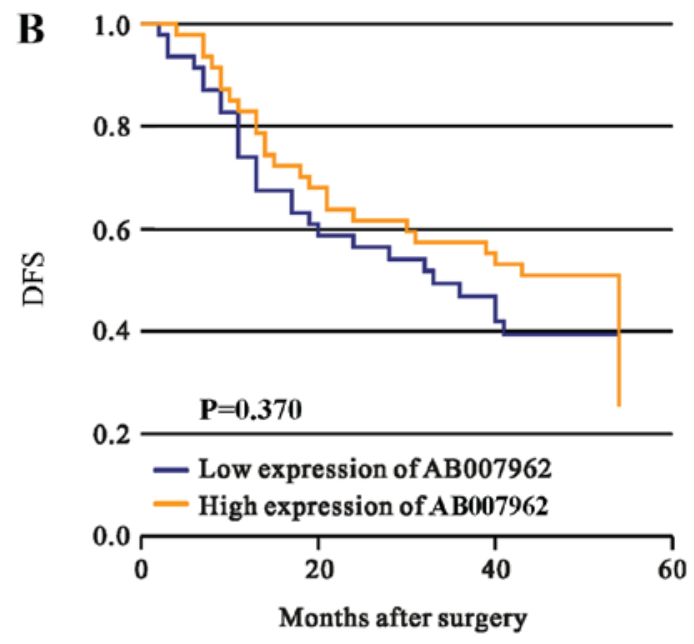

D

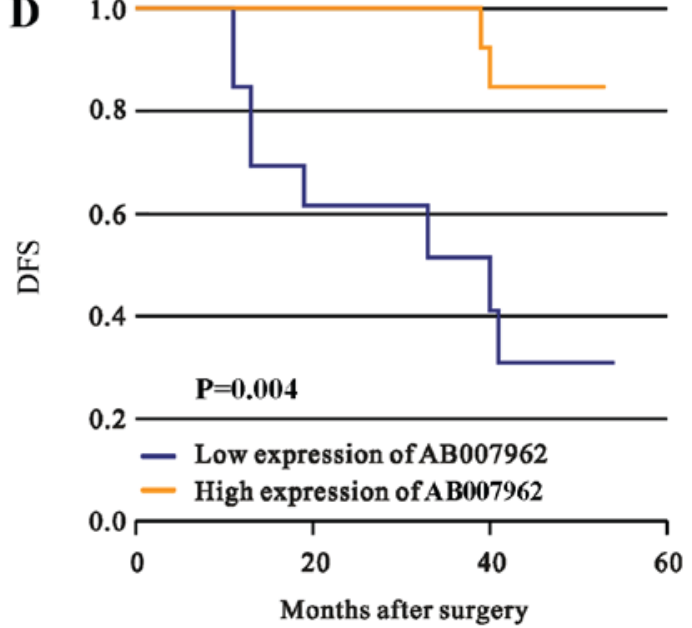

Figure 2. Kaplan-Meier survival curves of patients with GC based on AB007962 expression status. (A) OS of 96 patients with GC in the low and high expression groups. (B) DFS of 96 patients with GC in the low and high expression groups. (C) OS of 28 patients with intestinal-type GC in the low and high expression groups. Patients with lower AB007962 expression have shorter overall survival times compared with the higher AB007962 expression group. (D) DFS of 28 patients with intestinal-type GC in the low and high expression groups. Patients with lower AB007962 expression have shorter Disease-free survival times compared with the higher AB007962 expression group. OS, overall survival; DFS, disease free survival; GC, gastric cancer.

relative gene expression were further analyzed in patients with GC from the TCGA gene expression datasets, and the results demonstrated that there was no statistical differences of AB007962 expression in cancerous tissues, compared with NATs [read per kilobase of transcript per million mapped reads $(\mathrm{RPKM})_{\mathrm{GC}}, 0.379 \pm 0.078 ; \mathrm{RPKM}_{\mathrm{NATs}}, 0.400 \pm 0.095$; Wilcoxon test, $\mathrm{P}=0.633$ ].

Association between AB007962 levels in cancer tissues and clinicopathological factors in patients with GC. The association between the expression level of AB007962 and clinicopathological factors in 96 patients with GC was investigated, including sex, age, tumor size, tumor location, macroscopic type, histological grade and TNM stage. No significant associations were identified between AB007962, and sex $(\mathrm{P}=0.154)$, age $(\mathrm{P}=0.772)$, tumor location $(\mathrm{P}=0.515)$, tumor size $(\mathrm{P}=0.078)$ macroscopic type $(\mathrm{P}=0.241)$, histological differentiation $(\mathrm{P}=0.492)$, pathological $(\mathrm{p}) \mathrm{T}$ stage $(\mathrm{P}=0.756)$, $\mathrm{pN}$ stage $(\mathrm{P}=0.070)$, pTNM stage $(\mathrm{P}=0.978)$ or invasion into the lymphatic vessels $(\mathrm{P}=0.820)$. Furthermore, through the use of TCGA gene expression datasets, no significant associations were identified between AB007962 expression and clinicopathological factors, including sex $(\mathrm{P}=0.726)$, Lauren type $(\mathrm{P}=0.152)$, histological differentiation $(\mathrm{P}=0.532)$, pT stage $(\mathrm{P}=0.250)$, pN stage $(\mathrm{P}=0.472)$ or $\mathrm{pTNM}$ stage $(\mathrm{P}=0.092)$.

Association between AB007962 expression and patient survival time. In order to investigate whether the expression level of AB007962 was associated with the prognosis of the 96 patients with GC, survival data on the patients following gastrectomy was collected. The median ratio of relative AB007962 expression in the 96 patients with GC was used as the cut-off value to divide the 96 patients into groups with high and low AB007962 expression. Kaplan-Meier analysis and the log-rank test were used to evaluate the association between AB007962 expression, and OS and DFS. The results demonstrated that there was no significant difference in $\mathrm{OS}(\mathrm{P}=0.147$; Fig. 2A) or DFS ( $\mathrm{P}=0.370$; Fig. 2B) between the two groups.

However, among 28 patients with intestinal-type GC, those with increased AB007962 expression had significantly increased OS ( $\mathrm{P}=0.006$; Fig. $2 \mathrm{C})$ and DFS $(\mathrm{P}=0.004$; Fig. 2D) compared with the reduced expression group. The univariate survival analysis for OS and DFS indicated 
Table I. Association between the expression of long non-coding RNA AB007962 and the clinicopathological features in 96 patients with gastric cancer.

\begin{tabular}{|c|c|c|c|}
\hline Characteristics & No. of patients $(n=96)$ & Expression level $^{\mathrm{a}}$ & P-value \\
\hline Age, years & & & 0.772 \\
\hline$\leq 62$ & 48 & $0.87(0.49-1.32)$ & \\
\hline$>62$ & 48 & $0.78(0.44-1.18)$ & \\
\hline Sex & & & 0.151 \\
\hline Male & 70 & $0.71(0.49-1.13)$ & \\
\hline Female & 26 & $1.07(0.48-1.49)$ & \\
\hline Tumor size, $\mathrm{cm}$ & & & 0.078 \\
\hline$\leq 5.5$ & 50 & $0.93(0.49-1.49)$ & \\
\hline$>5.5$ & 46 & $0.70(0.50-1.04)$ & \\
\hline Tumor location & & & 0.515 \\
\hline Upper stomach & 5 & $0.41(0.25-1.46)$ & \\
\hline Middle stomach & 40 & $0.73(0.49-1.17)$ & \\
\hline Lower stomach & 47 & $0.92(0.49-1.35)$ & \\
\hline Entire stomach & 4 & $1.05(0.63-1.48)$ & \\
\hline Macroscopic type ${ }^{b}$ & & & 0.241 \\
\hline Borrmann I+II & 10 & $1.02(0.79-1.37)$ & \\
\hline Borrmann III+IV & 83 & $0.72(0.48-1.20)$ & \\
\hline Histological grade & & & 0.492 \\
\hline Good & 39 & $0.72(0.48-1.18)$ & \\
\hline Poor & 57 & $0.86(0.49-1.32)$ & \\
\hline Lauren grade & & & 0.693 \\
\hline Intestinal type & 28 & $0.78(0.52-1.40)$ & \\
\hline Diffuse type & 68 & $0.83(0.48-1.19)$ & \\
\hline pT stage & & & 0.953 \\
\hline $\mathrm{T}_{1}+\mathrm{T}_{2}$ & 13 & $0.73(0.45-1.34)$ & \\
\hline $\mathrm{T}_{3}+\mathrm{T}_{4}$ & 83 & $0.83(0.49-1.24)$ & \\
\hline pN stage & & & 0.070 \\
\hline N0 & 19 & $0.71(0.37-1.20)$ & \\
\hline N1 & 17 & $1.12(0.87-1.86)$ & \\
\hline N2 & 23 & $0.67(0.42-1.43)$ & \\
\hline N3 & 37 & $0.71(0.49-1.05)$ & \\
\hline pTNM stage & & & 0.977 \\
\hline I & 7 & $0.89(0.35-1.20)$ & \\
\hline II & 19 & $0.73(0.41-1.24)$ & \\
\hline III & 70 & $0.82(0.50-1.28)$ & \\
\hline Invasion into lymphatic vessels & & & 0.817 \\
\hline Negative & 50 & $0.73(0.49-1.21)$ & \\
\hline Positive & 46 & $0.86(0.49-1.41)$ & \\
\hline
\end{tabular}

${ }^{\mathrm{a} A B}$ 007962 median relative expression (25th-75th percentile); ${ }^{\mathrm{b}} 3$ patients had early-stage gastric cancer. TNM, Tumor-Node-Metastasis; p, pathological.

that $\mathrm{AB} 007962$ expression $(\mathrm{P}=0.016)$ and tumor location $(\mathrm{P}=0.026)$ were prognostic factors for $\mathrm{OS}$, and $\mathrm{AB} 007962$ expression $(\mathrm{P}=0.015)$ and tumor size $(\mathrm{P}=0.033)$ were significant prognostic factors for DFS (Table I). $\mathrm{P}<0.05$ was considered to indicate a statistically significant difference for multivariate Cox analysis, and the multivariate analysis confirmed that AB007962 expression levels were retained as an independent prognostic indicator for OS $(\mathrm{HR}=6.185$; 95\% CI, 1.331-28.732; $\mathrm{P}=0.020)$ and DFS $(\mathrm{HR}=7.982$; 95\% CI, 1.638-38.894; $\mathrm{P}=0.010$ ) (Table II) of patients with intestinal-type GC.

Nevertheless, from TCGA gene expression datasets, no significant association was identified between AB007962 expression, and the OS and DFS of patients with intestinal-type GC. 
Table II. Univariate and multivariate Cox regression analyses AB007962 for overall survival times or Disease-free survival times of patients with intestinal-type gastric cancer in the study cohort $(n=28)$.

\begin{tabular}{|c|c|c|c|c|c|c|}
\hline \multirow[b]{2}{*}{ Variables } & \multicolumn{3}{|c|}{ OS } & \multicolumn{3}{|c|}{ DFS } \\
\hline & HR & $95 \% \mathrm{CI}$ & P-value & HR & $95 \% \mathrm{CI}$ & P-value \\
\hline \multicolumn{7}{|l|}{ Univariate analysis } \\
\hline AB007962 expression (high vs. low) & 6.540 & $1.419-30.134$ & $0.016^{\mathrm{a}}$ & 7.044 & $1.469-33.784$ & $0.015^{\mathrm{a}}$ \\
\hline Tumor size $(\leq 5.5$ vs. $>5.5 \mathrm{~cm})$ & 0.557 & $0.178-1.743$ & 0.315 & 0.228 & $0.059-0.889$ & $0.033^{\mathrm{a}}$ \\
\hline Age ( $\leq 62$ vs. $>62$ years $)$ & 1.048 & $0.331-3.318$ & 0.936 & 1.271 & $0.367-4.396$ & 0.705 \\
\hline Sex (male vs. female) & 0.883 & $0.192-4.054$ & 0.872 & 0.595 & $0.075-4.708$ & 0.623 \\
\hline Tumor location (middle + lower vs. upper) & 5.711 & $1.234-2.436$ & $0.026^{\mathrm{a}}$ & 2.433 & $0.628-9.426$ & 0.198 \\
\hline Macroscopic type (Borrmann I+II vs. III+IV) & 0.689 & $0.143-3.329$ & 0.643 & 0.464 & $0.059-3.676$ & 0.467 \\
\hline Invasion depth (T1+T2 vs. T3+T4) & 0.427 & $0.055-3.314$ & 0.415 & 0.569 & $0.072-4.493$ & 0.592 \\
\hline Regional lymph nodes (N0 vs. N1+N2+N3) & 1.800 & $0.565-5.732$ & 0.320 & 1.727 & $0.482-6.188$ & 0.401 \\
\hline TNM stage (I+II vs. III+IV) & 1.340 & $0.430-4.177$ & 0.614 & 0.885 & $0.249-3.143$ & 0.850 \\
\hline Invasion into lymphatic vessels (negative vs. positive) & 1.497 & $0.449-4.986$ & 0.511 & 1.049 & $0.295-3.727$ & 0.941 \\
\hline \multicolumn{7}{|l|}{ Multivariate analysis } \\
\hline AB007962 expression (high vs. low) & 6.185 & $1.331-28.732$ & $0.020^{\mathrm{a}}$ & 7.982 & $1.638-38.894$ & $0.010^{\mathrm{a}}$ \\
\hline Tumor size $(\leq 5.5$ vs. $>5.5 \mathrm{~cm})$ & NA & NA & NA & 0.199 & $0.050-0.788$ & $0.022^{\mathrm{a}}$ \\
\hline Tumor location (middle + lower vs. upper) & 5.403 & $1.152-25.349$ & $0.032^{\mathrm{a}}$ & NA & NA & NA \\
\hline
\end{tabular}

${ }^{a} \mathrm{P}<0.05$ was considered to indicate a statistically significant difference. OS, overall survival; DFS, disease-free survival; CI, confidence interval; HR, hazard ratio; TNM, Tumor-Node-Metastasis; NA, not available.

\section{Discussion}

Currently, the majority of GC cases are diagnosed at an advanced stage and patients have a poor prognosis (18); thus, early diagnosis of GC is of great importance. In order to achieve early diagnosis of GC, novel biomarkers for diagnosis and prognosis should be determined and used in clinical medicine. For example, Japan and South Korea have provided a government-sponsored screening program for GC, which has contributed to a low mortality-to-incidence ratio in these countries (19).

Previously, numerous studies have demonstrated that aberrant expression of lncRNAs serves an essential role in various human cancer types during the process of initiation and development (20-22). Furthermore, increasing evidence indicates that a number of lncRNAs serve as novel diagnostic and prognostic biomarkers in GC $(9,13,23-25)$. For example, two well-known IncRNAs, H19 and maternally expressed 3 (MEG3), were reported to be dysregulated in GC $(5,26)$. H19 is overexpressed in GC tissues and plasma, compared with NATs (24); therefore, the level of H19 in plasma may be used to distinguish patients with early stage GC from healthy individuals. Additionally, its sensitivity is notably increased, compared with traditional biomarkers in plasma, including carcinoembryonic antigen and carbohydrate antigen-199; therefore, the expression of H19 may be used to predict the prognosis of patients with GC $(5,24,27)$. Furthermore, Sun et al (26) reported that MEG3 is significantly down-regulated and is associated with reduced OS times in GC, and therefore it also served as a prognostic biomarker for patients with GC. In the present study, the expression level of AB007962 was investigated in GC tissues and NATs by RT-qPCR. The result indicated that $\mathrm{AB} 007962$ was down-regulated in the majority of the $96 \mathrm{GC}$ tissues, compared with their matched NATs. These results indicated that AB0074962 may be used as a novel diagnostic biomarker in GC.

In a previous study, Zhang et al (8) determined that antisense non-coding RNA in the INK4 locus (ANRIL) was significantly associated with tumor size, and indicated that knockdown of ANRIL inhibited GC cell proliferation in vitro. Sun et al (26) reported, similarly, that MEG3 was associated with tumor size and it may also regulate cell proliferation in GC. In the present study, the expression levels of AB007962 decreased in patients with larger tumor size; therefore, it was hypothesized that low expression levels of AB007962 may contribute to the proliferation of GC. Whether AB007962 regulated cell proliferation should be investigated further in the future.

The Lauren classification dates back to 1965 (28), and it is frequently used in clinical diagnosis and treatment (29). According to the Lauren classification, GC may be divided into intestinal, diffuse and mixed types (28). A previous study indicated that patients with intestinal-type GC have an improved prognosis, compared with those with other Lauren types (30). The intestinal type of GC is described as a tumor with glandular architecture and resembling colonic carcinoma (31), and its relative frequency is $\sim 54 \%$ (32). A number of studies have reported that intestinal-type GC is frequently associated with intestinal metaplasia and Helicobacter pylori infection $(33,34)$.

Previously, researchers have determined that a number of biomarkers predict the prognosis of patients with GC of different Lauren types. Human epithelial growth factor receptor 2 (HER2) is a member of the HER family. Its overexpression has been frequently observed in intestinal-type GC (35). Qiu et al (31) 
determined that HER 2 positivity was an independent prognostic factor in patients with intestinal-type GC. Furthermore, it was reported that patients with HER2-negative and intestinal-types exhibit the longest OS and DFS times (31). HOTAIR may also act as a prognostic biomarker in diffuse-type GC, whereby patients with increased HOTAIR expression have a reduced prognosis compared with those with reduced HOTAIR expression (10). In the present study, it was determined that AB007962 expression was significantly associated with poor prognosis in 28 patients with intestinal-type GC, with patients with a reduced expression level of AB007962 having a reduced prognosis, compared with those with an increased expression level. These results indicated that AB0074962 may act as a novel prognostic biomarker in intestinal-type GC.

In the present study, it was determined that AB007962 expression has a negative association with tumor size. It was also negatively associated with the OS and DFS time of patients with intestinal-type GC; however, the analysis of TCGA dataset of 274 GC patients indicated no significant association between AB007962 expression, clinicopathological factors and patient survival time. The difference of results may be attributed to different population data and different measuring methods. Firstly, all patients in the present study were from the Han population living in North China. The majority of the patients in the TCGA dataset were non-Asian (73.36\%), with no patients from the Han population living in North China. Additionally, RT-qPCR was performed to measure the expression level of AB007962 in GC tissues and matched NATs; however, AB007962 expression levels were determined by RNA Sequencing in TCGA dataset. Future studies should expand the sample size and investigate if these data also apply to other populations.

In conclusion, the results of the present study indicated that AB007962 expression is downregulated in GC. Furthermore, the expression level of AB007962 may predict the outcome of patients with intestinal-type GC; therefore, AB007962 is a potential novel biomarker for prognosis. However, the function and molecular mechanisms of AB007962, particularly in cell proliferation, requires further study.

\section{Acknowledgements}

The authors would like to thank the Department of Surgical Oncology of The First Hospital of China Medical University for providing human gastric tissue samples. Additionally, the authors would like to thank the College of China Medical University for technical assistance in experiments.

\section{Funding}

The present study was supported by National Science Foundation of China (grant nos. 81201888, 81372549 and 81172370), Clinical Capability Construction Project for Liaoning Provincial Hospitals (grant no. LNCCC-A01-2014) and Natural Science Foundation of Liaoning Province (grant no. 2014029201).

\section{Availability of data and materials}

All data generated or analyzed during the present study are included in this published article.

\section{Authors' contributions}

YS and ZW designed experiments, JW and YY performed experiments, PG, XC and BM analyzed data, JW and YY wrote the manuscript, JS was responsible for interpreting the data and revising the article. All authors read and approved the final manuscript.

\section{Ethics approval and consent to participate}

The present study was approved by the Ethics Committee of the First Hospital of China Medical University. All participants provided written informed consent.

\section{Patient consent for publication}

Written informed consent was obtained from each patient.

\section{Competing interests}

The authors declare that they no competing interests.

\section{References}

1. Herszényi L and Tulassay Z: Epidemiology of gastrointestinal and liver tumors. Eur Rev Med Pharmacol Sci 14: 249-258, 2010.

2. Jemal A, Siegel R, Xu J and Ward E: Cancer statistics, 2010. CA Cancer J Clin 60: 277-300, 2010.

3. Kagawa S, Shigeyasu K, Ishida M, Watanabe M, Tazawa H, Nagasaka T, Shirakawa Y and Fujiwara T: Molecular diagnosis and therapy for occult peritoneal metastasis in gastric cancer patients. World J Gastroenterol 20: 17796-17803, 2014.

4. Esteller M: Non-coding RNAs in human disease. Nat Rev Genet 12: 861-874, 2011.

5. Li H, Yu B, Li J, Su L, Yan M, Zhu Z and Liu B: Overexpression of 1ncRNA H19 enhances carcinogenesis and metastasis of gastric cancer. Oncotarget 5: 2318-2329, 2014.

6. Liu XH, Sun M, Nie FQ, Ge YB, Zhang EB, Yin DD, Kong R, Xia R, Lu KH, Li JH, et al: Lnc RNA HOTAIR functions as a competing endogenous RNA to regulate HER 2 expression by sponging miR-331-3p in gastric cancer. Mol Cancer 13: 92, 2014.

7. Xu TP, Liu XX, Xia R, Yin L, Kong R, Chen WM, Huang MD and Shu YQ: SP1-induced upregulation of the long noncoding RNA TINCR regulates cell proliferation and apoptosis by affecting KLF2 mRNA stability in gastric cancer. Oncogene 34: 5648-5661, 2015.

8. Zhang EB, Kong R, Yin DD, You LH, Sun M, Han L, Xu TP, Xia R, Yang JS, De W and Chen Jf: Long noncoding RNA ANRIL indicates a poor prognosis of gastric cancer and promotes tumor growth by epigenetically silencing of $\mathrm{miR}-99 \mathrm{a} / \mathrm{miR}-449 \mathrm{a}$. Oncotarget 5: 2276-2292, 2014.

9. Xu ZY, Yu QM, Du YA, Yang LT, Dong RZ, Huang L, Yu PF and Cheng XD: Knockdown of long non-coding RNA HOTAIR suppresses tumor invasion and reverses epithelial-mesenchymal transition in gastric cancer. Int J Biol Sci 9: 587-597, 2013.

10. Endo H, Shiroki T, Nakagawa T, Yokoyama M, Tamai K, Yamanami H, Fujiya T, Sato I, Yamaguchi K, Tanaka N, et al: Enhanced expression of long non-coding RNA HOTAIR is associated with the development of gastric cancer. PLoS One 8: e77070, 2013

11. Yang F, Bi J, Xue X, Zheng L, Zhi K, Hua J and Fang G: Up-regulated long non-coding RNA H19 contributes to proliferation of gastric cancer cells. FEBS J 279: 3159-3165, 2012.

12. Zhuang M, Gao W, Xu J, Wang P and Shu Y: The long non-coding RNA H19-derived miR-675 modulates human gastric cancer cell proliferation by targeting tumor suppressor RUNX1. Biochem Biophys Res Commun 448: 315-322, 2014.

13. Zhang EB, Han L, Yin DD, Kong R, De W and Chen J: c-Myc-induced, long, noncoding $\mathrm{H} 19$ affects cell proliferation and predicts a poor prognosis in patients with gastric cancer. Med Oncol 31: 914, 2014. 
14. Hamilton SR and Aaltonen LA (eds): World Health Organization Classifcation of Tumours. Pathology and genetics of tumours of the digestive system. International Agency for Research on Cancer (IARC) Press, Lyon, Paris, 2000.

15. Sobin LH, Gospodarowicz M and Wittekind C (eds): International Union Against Cancer (UICC). TNM classifcation of malignant tumours. 7th edition. Hoboken, NJ: Wiley-Liss, New York, NY, USA, pp117-126, 2010.

16. Livak KJ and Schmittgen TD: Analysis of relative gene expression data using real-time quantitative PCR and the 2(-Delta Delta C(T)) method. Methods 25: 402-408, 2001.

17. Mortazavi A, Williams BA, McCue K, Schaeffer L and Wold B: Mapping and quantifying mammalian transcriptomes by RNA-Seq. Nat Methods 5: 621-628, 2008.

18. Thiel A and Ristimäki A: Gastric cancer: Basic aspects. Helicobacter 17 (Suppl 1): S26-S29, 2012.

19. Shen L, Shan YS, Hu HM, Price TJ, Sirohi B, Yeh KH, Yang YH, Sano T, Yang HK, Zhang X, et al: Management of gastric cancer in Asia: Resource-stratified guidelines. Lancet Oncol 14: e535-e547, 2013

20. Mercer TR, Dinger ME and Mattick JS: Long non-coding RNAs: Insights into functions. Nat Rev Genet 10: 155-159, 2009.

21. Hu W, Alvarez-Dominguez JR and Lodish HF: Regulation of mammalian cell differentiation by long non-coding RNAs. EMBO Rep 13: 971-983, 2012.

22. Kaikkonen MU, Lam MT and Glass CK: Non-coding RNAs as regulators of gene expression and epigenetics. Cardiovasc Res 90: 430-440, 2011.

23. Lee NK, Lee JH, Park CH, Yu D, Lee YC, Cheong JH, Noh SH and Lee SK: Long non-coding RNA HOTAIR promotes carcinogenesis and invasion of gastric adenocarcinoma. Biochem Biophys Res Commun 451: 171-178, 2014.

24. Zhou X, Yin C, Dang Y, Ye F and Zhang G: Identification of the long non-coding RNA H19 in plasma as a novel biomarker for diagnosis of gastric cancer. Sci Rep 5: 11516, 2015.

25. Kretz M, Siprashvili Z, Chu C, Webster DE, Zehnder A, Qu K Lee CS, Flockhart RJ, Groff AF, Chow J, et al: Control of somatic tissue differentiation by the long non-coding RNA TINCR. Nature 493: 231-235, 2013.
26. Sun M, Xia R, Jin F, Xu T, Liu Z, De W and Liu X: Downregulated long noncoding RNA MEG3 is associated with poor prognosis and promotes cell proliferation in gastric cancer. Tumour Biol 35: 1065-1073, 2014

27. Arita T, Ichikawa D, Konishi H, Komatsu S, Shiozaki A, Shoda K, Kawaguchi T, Hirajima S, Nagata H, Kubota T, et al: Circulating long non-coding RNAs in plasma of patients with gastric cancer. Anticancer Res 33: 3185-3193, 2013.

28. Lauren P: The two histological main types of gastric carcinoma: Diffuse and so-called intestinal-type carcinoma. An attempt at a histo-clinical classification. Acta Pathol Microbiol Scand 64: 31-49, 1965

29. Hu B,ElHajj N, Sittler S, Lammert N, Barnes R and Meloni-Ehrig A: Gastric cancer: Classification, histology and application of molecular pathology. J Gastrointest Oncol 3: 251-261, 2012.

30. Bravo Neto GP, dos Santos EG, Victer FC and Carvalho CE: Lymph node metastasis in early gastric cancer. Rev Col Bras Cir 41: 11-17, 2014.

31. Qiu M, Zhou Y, Zhang X, Wang Z, Wang F, Shao J, Lu J, Jin Y, Wei X, Zhang D, et al: Lauren classification combined with HER2 status is a better prognostic factor in Chinese gastric cancer patients. BMC Cancer 14: 823, 2014.

32. Polkowski W, van Sandick JW, Offerhaus GJ, ten Kate FJ, Mulder J, Obertop H and van Lanschot JJ: Prognostic value of Laurén classification and c-erbB-2 oncogene overexpression in adenocarcinoma of the esophagus and gastroesophageal junction. Ann Surg Oncol 6: 290-297, 1999.

33. Kaneko S and Yoshimura T: Time trend analysis of gastric cancer incidence in Japan by histological types, 1975-1989. Br J Cancer 84: 400-405, 2001.

34. Parsonnet J, Vandersteen D, Goates J, Sibley RK, Pritikin J and Chang Y: Helicobacter pylori infection in intestinal-and diffuse-type gastric adenocarcinomas. J Natl Cancer Inst 83: 640-643, 1991.

35. Bang YJ, Van Cutsem E, Feyereislova A, Chung HC, Shen L, Sawaki A, Lordick F, Ohtsu A, Omuro Y, Satoh T, et al: Trastuzumab in combination with chemotherapy versus chemotherapy alone for treatment of HER2-positive advanced gastric or gastro-oesophageal junction cancer (ToGA): A phase 3, open-label, randomised controlled trial. Lancet 376: 687-697, 2010. 